\title{
Simulations of a multipactor-inhibited waveguide geometry
}

\author{
Eric Chojnacki \\ Laboratory of Nuclear Studies, Cornell University, Ithaca, New York 14853
}

(Received 21 January 2000; published 20 March 2000)

\begin{abstract}
Studies of multipactor resonances in coaxial and hollow waveguide via standard particle trajectory and secondary emission numerical simulation techniques are presented. Agreement between simulations and experimentally observed multipactor barriers gives confidence in results of simulation studies of a wedge-shaped hollow waveguide geometry that shows promise of being far less susceptible to multipactor resonances than standard coax and hollow rectangular waveguides.
\end{abstract}

PACS numbers: 52.80.Vp, 52.80.Pi, 52.20.Dq

\section{INTRODUCTION}

Multipactor resonances in the waveguide feeding $\mathrm{rf}$ cavities often hinder coupling of rf power to particle beams. This is especially true in superconducting rf (SRF) cavities with characteristic long-pulse or $\mathrm{cw}$ operation, exacerbated by cold waveguide regions containing condensed gases which enhance the surface secondary emission coefficient. Previous numerical simulation studies of multipactor in waveguide have reported good agreement between simulated power barriers and those experimentally observed. Waveguide geometries studied have included the coaxial waveguide of the TESLA Test Facility (TTF) $[1,2]$ and hollow rectangular waveguide of the Cornell Electron Storage Ring (CESR) [3]. This lends confidence to the predictive power of such simulation studies.

A clue to suppressing multipactor in waveguide is provided by the experience of suppressing many virulent multipactor resonances in cavities. This was accomplished over two decades ago by implementation of a rounded SRF cavity profile instead of pillbox [4]. Electron trajectory tracking codes subsequently quantified the benefits of a rounded cavity profile in regard to eliminating many multipactor resonances [5]. Thus, a similar geometrical approach to waveguide coupler variation is presented here. First, the salient features of a multipactor simulation code will be discussed. Results from the code will then be presented for familiar multipactor resonances in coaxial and hollow waveguide with particular attention to multipactor trajectory characteristics. Insight from these familiar resonances will then be used to propose a multipactor-inhibited wedge-shaped hollow waveguide geometry. The simulation code will then be applied to this "wedgeguide" and the promising results summarized.

\section{MULTIPACTOR SIMULATION CODE}

The simulation code utilized here employs many wellknown procedures for predicting multipactor resonances [6]. Electron trajectories were computed by fourth order Runge-Kutta solution of the full 3D Lorentz force equations. All electromagnetic fields for the cases in this study were single modes of axially uniform waveguide which were expressible in analytic form. Further, the modes were taken as simple cw traveling waves. Electrons were launched from a range of selected cross-sectional locations at a range of $\mathrm{rf}$ phases, typically from $-30^{\circ}$ to $180^{\circ}$ in $5^{\circ}$ steps with the electric field $E \propto \sin (\phi)$. rf power was scanned over a selected range in $1 \mathrm{~kW}$ steps. The initial electron and all secondaries were launched at an energy of $2 \mathrm{eV}$ normal to the surface. The code typically runs for 20 impacts per initial launch condition, terminated if secondary multiplication becomes $<10^{-3}$ or if the impacting electron energy is below some threshold, such as $0.1 \mathrm{eV}$. For each case of rf power level, launch phase, and launch location, secondary electron multiplication due to wall impacts is logged by a multiplicative counter utilizing a familiar curve of secondary electron yield versus impact energy [7]. At each power level these secondary multiplications are summed over all launch phases. This is similar to what has been termed an "enhanced counter function," except there is no termination of the simulation decided by electric field polarity upon secondary launch [6].

Electron trajectories in multipactor simulations can be sensitive to numerical error due to the large number of integration time steps [8]. For the cases considered here with $500 \mathrm{MHz}$ rf frequency and peak electric field of the order $100 \mathrm{kV} / \mathrm{m}$, the optimal time step was found to be of the order 1 ps. Multipactor resonant trajectories were fairly tolerant to numerical error, yielding nearly identical results in regard to final trajectory position and secondary multiplication for time steps anywhere in the range 0.1-10 ps. Nonresonant trajectories were quite sensitive to numerical error, even occasionally showing false resonances that were elucidated by evaluation of accumulated error and trajectory reproducibility upon modest variation in time step.

\section{COAXIAL AND HOLLOW RECTANGULAR WAVEGUIDE}

As a test of the code described in Sec. II, it was run for known simulated and experimental cases of coaxial and 
rectangular waveguide. The results of secondary electron multiplication over a range of $\mathrm{rf}$ power are shown in Fig. 1 for TESLA $40 \mathrm{~mm}, 50 \Omega$ coax driven at $1.3 \mathrm{GHz}$ and in Fig. 2 for CESR $10.2 \mathrm{~cm} \times 43.2 \mathrm{~cm}$ rectangular waveguide driven at $500 \mathrm{MHz}$. Both Fig. 1 and Fig. 2 correspond to secondary multiplication from a single initial launch location as described in the next two paragraphs. The multipactor barriers represented in Figs. 1 and 2 agree well with previous simulations and experiments $[2,3]$.

In rectangular waveguide, $\mathrm{TE}_{10}$ mode two-point multipactor resonances occur between broad walls along the cross-sectional midline at the peak of the electric field.
Using the rectangular waveguide $\mathrm{TE}_{10}$ field convention of $E_{y}, B_{x}$, and $B_{z}$, these resonances have slow migration along the $z$ axis arising from the $v_{y} \times B_{x}$ Lorentz force. Interestingly, trajectories launched displaced from the midline experience a transverse $v_{y} \times B_{z}$ force and migrate to the sidewall after a few impacts, as shown in Fig. 3. There, the electric field is zero and the magnetic field finite, thus successive cyclic low energy impacts quickly damp secondary multiplication to negligible levels. Even a small perturbation from the midline equilibrium, such as $1 \mathrm{~mm}$, leads to migration to the sidewall, as shown in Fig. 4. This implies that a narrow strip along

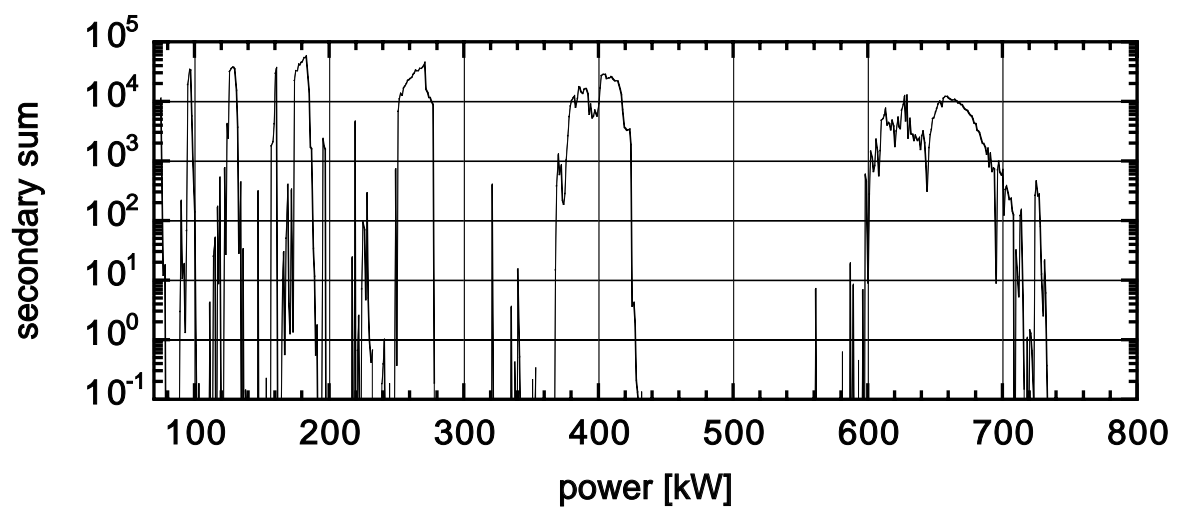

FIG. 1. Secondary multiplication summed over all launch phases in TESLA $40 \mathrm{~mm}, 50 \Omega$ coax driven at $1.3 \mathrm{GHz}$ with a maximum of 20 impacts from a single initial launch location on the outer conductor.

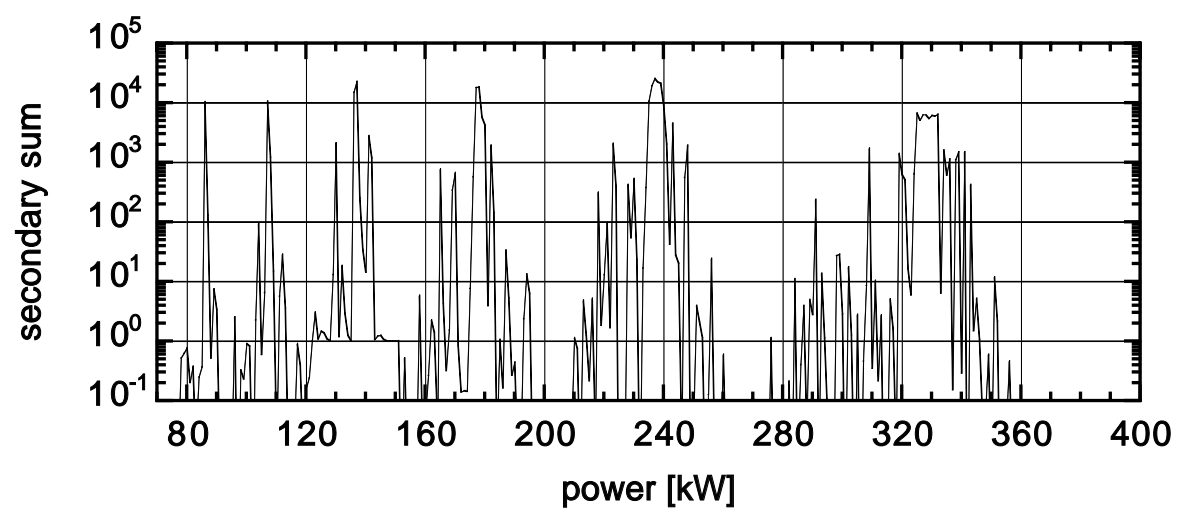

FIG. 2. Secondary multiplication summed over all launch phases in CESR $10.2 \mathrm{~cm} \times 43.1 \mathrm{~cm}$ rectangular waveguide driven at $500 \mathrm{MHz}$ with a maximum of 20 impacts from a single initial launch location on the midline.

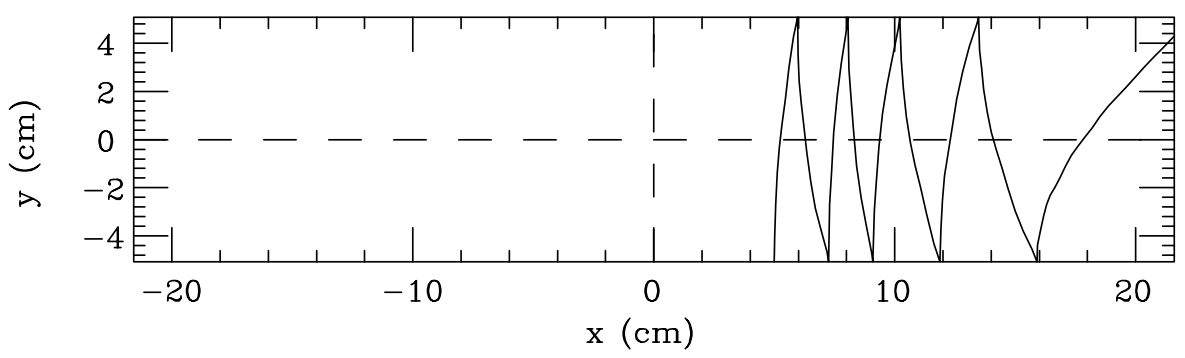

FIG. 3. Trajectory of an electron launched $5 \mathrm{~cm}$ from the midline in CESR rectangular waveguide propagating the TE 10 mode. 


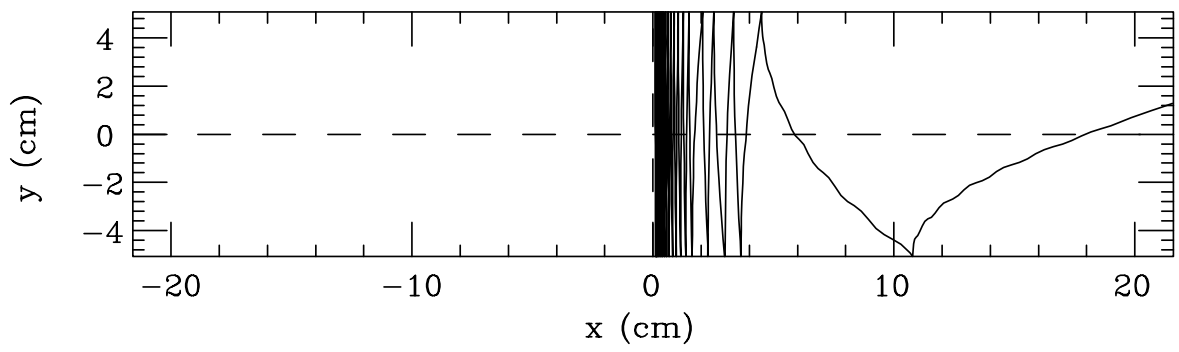

FIG. 4. Trajectory of an electron launched $1 \mathrm{~mm}$ from the midline in CESR rectangular waveguide propagating the TE 10 mode.

the center of rectangular waveguide is solely responsible for observed multipactor barriers. Though seemingly surprising, the barriers presented in Fig. 2 by such midline trajectories agree quite well with experiment.

For coaxial couplers with aligned, round conductors, troublesome multipactor resonances driven by the axisymmetric TEM mode typically occur with repeating impacts on the outer conductor, independent of azimuthal launch location. Trajectories closely follow the radial electric field and have slow migration along the axis arising from the $v_{r} \times B_{\theta}$ Lorentz force. Again, coax multipactor barrier power levels predicted by simulation agree well with experiment.

\section{WEDGEGUIDE}

Since both rectangular and coaxial waveguide multipactor trajectories remain well aligned with the respective mode's electric field, a resonance-disrupting effect may be provided by altering the waveguide cross section so as to force curvature on the electric field. This could disallow trajectories from traversing repeatable paths and even guide electrons to regions of zero electric field.

One such unconventional waveguide cross section is illustrated in Fig. 5. Rectangular waveguide is altered to have the broad walls nonparallel and the sidewalls as circular arcs to facilitate analysis. This "wedgeguide" has a lowest-cutoff mode similar to the rectangular $\mathrm{TE}_{10}$. Modifications to coax have been explored elsewhere, but since TEM modes have no region of zero electric field and topology requires at least two places where inner and outer conductor surface normal vectors point at each other, resonant trajectories remained $[9,10]$. Discussion of the wedge-shaped hollow waveguide follows.

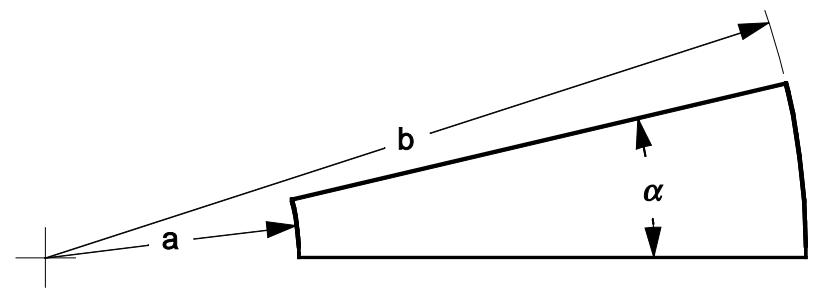

FIG. 5. Wedge-shaped cross section of a hollow waveguide.

\section{A. Wedgeguide fundamental mode}

A MAFIA [11] solution of the electric field of the lowestcutoff mode of the waveguide illustrated in Fig. 5 is shown in Fig. 6, having a field pattern analogous to that of the rectangular $\mathrm{TE}_{10}$. This mode can be analytically treated in cylindrical coordinates as a higher order TE mode of coaxial waveguide [12]. The broad walls are on $\theta=$ const surfaces and the sidewalls are on $r=$ const surfaces, as indicated in Fig. 5 by the dimensions $a$, $b$, and $\alpha$. The azimuthal harmonic of this TE mode is $n=0$, and the cutoff wave number $k_{c}$ is given by solution of the transcendental equation

$$
\frac{J_{0}^{\prime}\left(k_{c} a\right)}{J_{0}^{\prime}\left(k_{c} b\right)}=\frac{N_{0}^{\prime}\left(k_{c} a\right)}{N_{0}^{\prime}\left(k_{c} b\right)}
$$

where $J_{n}$ and $N_{n}$ are Bessel functions of the first and second kind, respectively. The field components are given by

$$
\begin{aligned}
& E_{\theta}=E_{0}\left[J_{1}\left(k_{c} r\right)-g N_{1}\left(k_{c} r\right)\right] e^{j(\omega t-\beta z)}, \\
& H_{r}=\frac{-\beta E_{\theta}}{\omega \mu}, \\
& H_{z}=\frac{-j k_{c} E_{0}}{\omega \mu}\left[J_{0}\left(k_{c} r\right)-g N_{0}\left(k_{c} r\right)\right] e^{j(\omega t-\beta z)},
\end{aligned}
$$

where

$$
g \equiv \frac{J_{1}\left(k_{c} a\right)}{N_{1}\left(k_{c} a\right)} .
$$

The peak azimuthal electric field occurs at a radius $r_{e}$ solving

$J_{0}\left(k_{c} r_{e}\right)-\frac{J_{1}\left(k_{c} r_{e}\right)}{k_{c} r_{e}}-g\left[N_{0}\left(k_{c} r_{e}\right)-\frac{N_{1}\left(k_{c} r_{e}\right)}{k_{c} r_{e}}\right]=0$,

and has the value

$$
E_{\theta \text { peak }}=E_{0}\left[J_{1}\left(k_{c} r_{e}\right)-g N_{1}\left(k_{c} r_{e}\right)\right] .
$$

Integration of the Poynting vector gives the propagating power as

$$
P=\frac{\alpha E_{0}^{2} \beta}{4 \omega \mu}\left\{r^{2}\left[R_{1}^{2}\left(k_{c} r\right)-R_{0}\left(k_{c} r\right) R_{2}\left(k_{c} r\right)\right]\right\}_{a}^{b},
$$




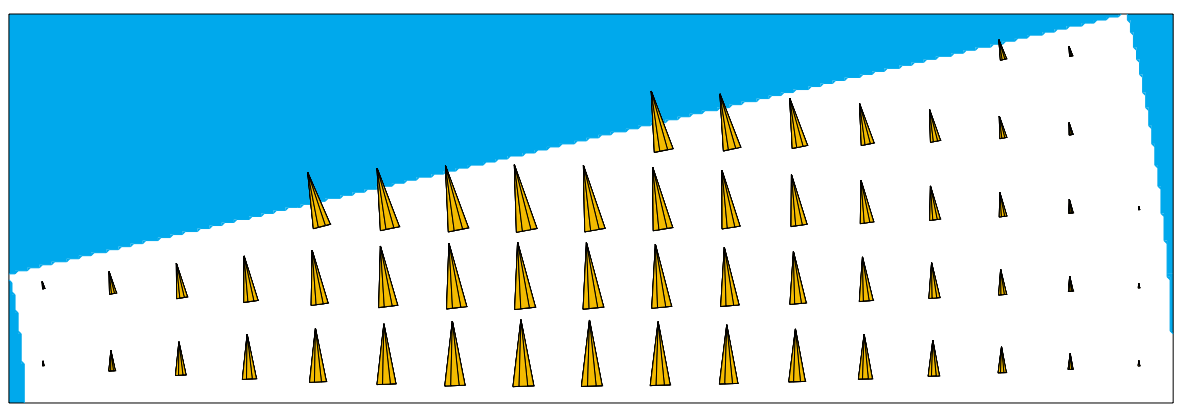

FIG. 6. (Color) MAFIA solution of the lowest-cutoff mode electric field in the wedge-shaped waveguide illustrated in Fig. 5, having a field pattern analogous to that of the rectangular $\mathrm{TE}_{10}$.

where

$$
R_{n}(x) \equiv J_{n}(x)-g N_{n}(x) .
$$

The analytic forms allow greater accuracy and computation speed of electron trajectories than using, e.g., interpolation of MAFIA fields shown in Fig. 6.

\section{B. Wedgeguide multipactor simulations}

The traveling-wave rf fields in Eqs. (2) were used in the above described multipactor code. The wedgeguide dimensions were taken as a modification to the CESR $10.2 \mathrm{~cm} \times 43.2 \mathrm{~cm}$ rectangular waveguide where one sidewall is effectively reduced to a $5.1 \mathrm{~cm}$ height and the other increased to $15.2 \mathrm{~cm}$ by having (Fig. 5) dimensions of $a=21.6 \mathrm{~cm}, b=64.8 \mathrm{~cm}, \alpha=13.2^{\circ}$, and again driven at $500 \mathrm{MHz}$.

Running simulations over comprehensive ranges of $\mathrm{rf}$ power, launch phase, and launch position in wedgeguide has revealed three characteristics of electron trajectories.

(i) The great majority of trajectories alternately impact on the broad walls and quickly become antiresonant with the rf. After a dozen or so impacts, the accumulated secondaries perform successive low energy impacts, and secondary multiplication quickly decays to negligible values.

(ii) Trajectories have a migration bias toward the larger radius sidewall due to the $E_{\theta}$ field curvature. Trajectories that survive antiresonant conditions well enough to maintain a significant secondary multiplication typically migrate to this wall. There, the vanishing electric and finite magnetic fields allow only successive cyclic low energy impacts that quickly damp secondary multiplication to negligible levels, analogous to trajectories displaced from the midline in normal rectangular waveguides.

(iii) The outward radial migration due to the $E_{\theta}$ field curvature opposes the net inward radial $v_{\theta} \times B_{z}$ force that occurs at radii less than the peak of the $E_{\theta}$ field as given by Eq. (4). For a range of trajectory radii there are specific rf power levels at which these opposing forces almost balance, and the electrons can remain close to resonant in an $r=$ const vicinity. Fortunately, the force balance is not constant along the trajectory nor on average, and the electrons eventually become antiresonant and/or migrate to a sidewall, in either case, ultimately experiencing successive low energy impacts that quickly damp secondary multiplication to negligible levels. An instance of this is shown in the trajectory in Fig. 7, wherein secondary multiplication temporarily increased by a factor of 100 before the trajectory became antiresonant and performed low energy impacts to reduce secondary multiplication to $<10^{-3}$.

Thus, among the multitude of power, launch phase, and launch position cases simulated for multipactor resonance in wedgeguide, none have exhibited a resonance which would present a multipactor power barrier. This is

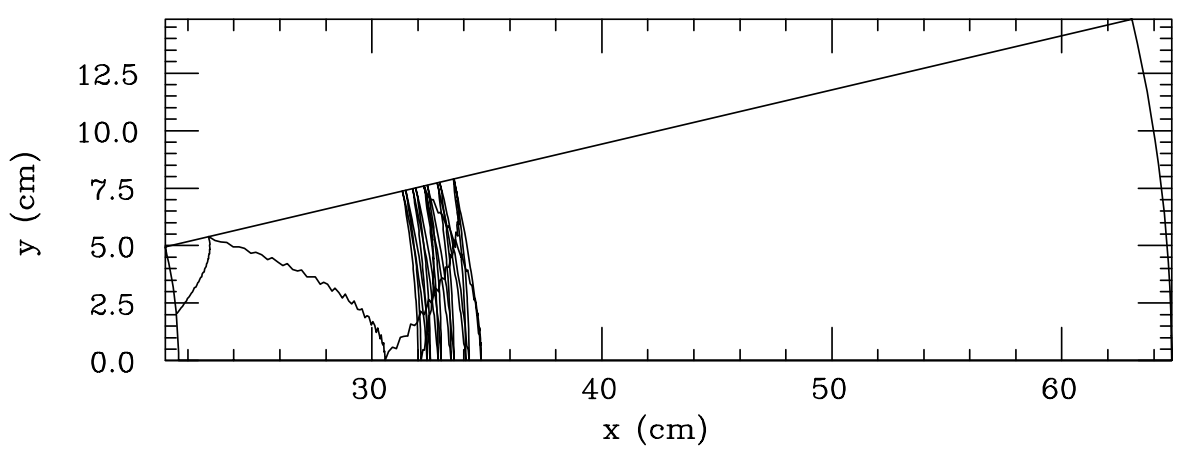

FIG. 7. Trajectory of an electron launched at a radius of $32 \mathrm{~cm}$ in wedgeguide and temporarily experiencing almost balanced transverse forces, eventually migrating to a sidewall and performing successive low energy impacts to reduce secondary multiplication to $<10^{-3}$. 


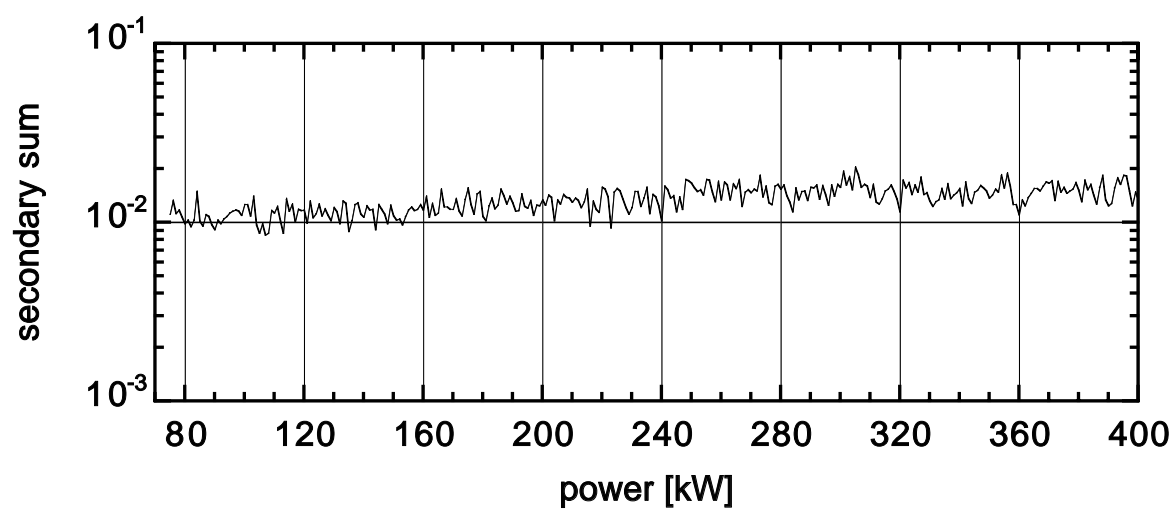

FIG. 8. Secondary multiplication summed over all launch phases in wedgeguide with dimensions $a=21.6 \mathrm{~cm}, b=64.8 \mathrm{~cm}$, $\alpha=13.2^{\circ}$, driven at $500 \mathrm{MHz}$, and initial launch radius at $29 \mathrm{~cm}$.

quantitatively illustrated in Fig. 8 where secondary multiplication versus rf power in wedgeguide is plotted for initial electron launch at a radius of $29 \mathrm{~cm}$ per the lower axis in Fig. 7. Launches at $29 \mathrm{~cm}$ have shown the greatest likelihood of exhibiting short term balanced trajectories, but, as explained in (iii) above, all such trajectories terminate in successive low energy impacts to yield secondary multiplication values embedded in Fig. 8 that are indistinguishable from those of completely antiresonant trajectories. Cases of secondary multiplication in wedgeguide for electron launch radii from 25 to $42 \mathrm{~cm}$ scanned in $0.5 \mathrm{~cm}$ steps are quite similar to Fig. 8, having values at all powers considerably less than unity. This is in sharp contrast to rectangular and coaxial waveguide which have numerous multipactor resonances yielding secondary multiplication of $>10^{4}$ after 20 impacts from a single launch location, as shown in Figs. 1 and 2.

\section{CONCLUSIONS}

Electron trajectory simulations show the "wedgeguide" waveguide geometry to be promising in having greatly reduced multipactor susceptibility, no resonances being found after simulating a multitude of cases of $\mathrm{rf}$ power, launch phase, and launch position. From an operational point of view, a waveguide coupler fabricated with this shape would still likely require processing to burn off emitters and "scrub" the waveguide surfaces. But it is hoped that such processing will proceed quickly and not show degradation as experienced with current cryogenic couplers. Care would also have to be taken to ensure there are a minimum of other multipactor-susceptible components in the coupler chain, such as developing a novel vacuum window comprised of a familiar self-matched elliptical ceramic [13], but framed in wedgeguide.

\section{ACKNOWLEDGMENTS}

I am grateful for useful discussions with R.L. Geng, J. Knobloch, and H. Padamsee.
[1] E. Somersalo, P. Yla-Oijala, and D. Proch, in Proceedings of the 1995 Particle Accelerator Conference, Dallas, Texas, edited by Laurie Gennari (IEEE, Piscataway, NJ, 1996), p. 1500.

[2] B. Dwersteg, in Proceedings of the 8th Workshop on RF Superconductivity, Padova, Italy, 1997, edited by V. Palmierii and A. Lombardi (LNL-INFN Report No. 133/98, 1998), p. 740.

[3] R. L. Geng and H. Padamsee, in Proceedings of the 1999 Particle Accelerator Conference, New York, edited by A. Luccio and W. MacKay (IEEE, Piscataway, NJ, 1999), p. 429.

[4] V. Lagomarsino, G. Manuzio, R. Parodi, and R. Vaccarone, IEEE Trans. Magn. 1, 25 (1979).

[5] U. Klein and D. Proch, in Proceedings of the Conference on Future Possibilities for Electron Accelerators, Charlottesville, Virginia, 1979, edited by J.S. McCarthy and R. R. Whitney (University of Virginia, Charlottesville, VA, 1979), p. N1.

[6] E. Somersalo, P. Yla-Oijala, D. Proch, and J. Sarvas, Part. Accel. 59, 107 (1998).

[7] H. Piel, in Proceedings of the CERN Accelerator School: Superconductivity in Particle Accelerators, Haus Rissen, Hamburg, Germany, 1988, edited by S. Turner (CERN, Geneva, 1989), p. 176.

[8] W.H. Press, B.P. Flannery, S. A. Teukolsky, and W. T. Vetterling, Numerical Recipes in Fortran 77: The Art of Scientific Computing (Cambridge University Press, Cambridge, England, 1996), 2nd ed., Vol. 1, p. 708.

[9] J. Tuckmantel, CERN LEP2 Note No. 94-25, 1994.

[10] J. Tuckmantel, in Proceedings of the 1995 Particle Accelerator Conference, Dallas, Texas (Ref. [1]), p. 1642.

[11] Computer code MAFIA, CST-Gesellschaft fur ComputerSimulationstechnik mbH; www.cst.de.

[12] S. Ramo, J. R. Whinnery, and T. Van Duzer, Fields and Waves in Communication Electronics (Wiley, New York, 1994), 3rd ed., p. 433.

[13] E. Chojnacki, T. Hays, J. Kirchgessner, H. Padamsee, M. Cole, and T. Schulteiss, in Proceedings of the 1997 Particle Accelerator Conference, Vancouver, Canada, edited by M. Comyn, M. K. Craddock, M. Reiser, and J. Thomson (IEEE, Piscataway, NJ, 1998), p. 3177. 